\title{
Mechanisms of Postsynaptic Plasticity: Remodeling of the Junctional Acetylcholine Receptor Cluster Induced by Motor Nerve Terminal Outgrowth
}

\author{
Woon Chee Yee and Alan Pestronk \\ Department of Neurology, Johns Hopkins University, School of Medicine, Baltimore, Maryland 21205
}

\begin{abstract}
Motor nerve terminal outgrowth (NTO) at neuromuscular junctions (NMJs) occurs rapidly in response to denervation changes in muscle. We have previously found that NTO can produce an elongation of the synaptic area of the NMJ as defined by cholinesterase-silver staining. In the present study, we examined the effects of NTO on a postsynaptic muscle membrane component, the usually stable cluster of acetylcholine receptors (AChRs) at the NMJ. NTO was evoked in rat soleus muscles using botulinum toxin. AChRs were demonstrated using immunocytochemistry or autoradiography of $\alpha$-bungarotoxin binding. Our results show that NTO induces rapid elongation of the cluster of AChRs at the NMJ within $7 \mathrm{~d}$ of treatment with botulinum toxin. The growth in the size of the AChR clusters was accompanied by an increase in the number of AChRs/NMJ. No elongation of AChR clusters was seen following surgical denervation, suggesting that cluster growth is related to NTO and not to denervation changes in muscle per se. Growth of NMJ-AChR clusters appeared to result primarily from 2 processes: insertion of new AChRs into the NMJ membrane and, surprisingly, redistribution of preexisting NMJ-AChRs. These results show that NTO can cause rapid changes in the normally stable cluster of AChRs at the NMJ. Motor nerve terminals provide a strong and anatomically precise control of AChRs at the NMJ.
\end{abstract}

The distribution and turnover of acctylcholine receptors (AChRs) in skeletal muscle membrane are regulated to a large extent by motor nerves. During development, AChRs cluster at the site of contact between the motor nerve terminal and the muscle membrane (Anderson and Cohen, 1977; Anderson et al., 1977; Bevan and Steinbach, 1977; Burden 1977; Braithwaite and Harris 1979; Frank and Fischbach, 1979). In innervated adult muscles, AChRs are localized almost exclusively at neuromuscular junctions (NMJs) (Miledi, 1960; Fambrough and Hartzell, 1972; Fertuck and Salpeter, 1976; Fambrough, 1979). The nerve induces considerable stability in these clusters of junctional AChRs,

\footnotetext{
Received May 23, 1986; revised Oct. 13, 1986; accepted Jan. 8, 1987.

This work was supported by Grants P01 NS19020 and 5R01 HD04817 from the National Institutes of Health, and U.S. Army Contract No. DAMD 17-85-C5069. W-C.Y. was supported in part by a Research Fellowship from the Muscular Dystrophy Association. We are grateful to Drs. Daniel B. Drachman and Elis F. Stanley for helpful suggestions on the experiments and to Ralph W. Kuncl for comments on the manuscript. We also thank Robert N. Adams, Mary E. Dorman, and Marilyn J. Peper for technical assistance, and Christine F. Salemi for assistance in the preparation of the manuscript.

Correspondence should be addressed to Alan Pestronk, M.D., Department of Neurology, Johns Hopkins School of Medicine, 600 N. Wolfe Street, Baltimore, MD 21205.

Copyright ( 1987 Society for Neuroscience $0270-6474 / 87 / 072019-06 \$ 02.00 / 0$
}

a property that differentiates them from AChRs appearing in the membrane of immature or denervated muscle (Berg and Hall, 1975; Chang and Huang, 1975; Axelrod et al., 1979; Fambrough, 1979; Levitt and Salpeter, 1981; Young and Poo, 1983; Stya and Axelrod, 1984).

In the course of recent studies of synaptic plasticity, we have found that outgrowth from motor nerve terminals can induce rapid modifications in previously stable structures at the NMJ (Pestronk et al., 1976; Pestronk and Drachman 1978b, 1985a). During the process of nerve terminal outgrowth (NTO) (e.g., after botulinum treatment), the arca of synaptic contact between motor nerve terminals and muscle fibers becomes elongated over periods as short as 5-7 d. We have shown that this change is accompanied by an increase in the length of the patch of cholinesterase at the NMJ (Pestronk and Drachman, 1978b). In the present experiments we asked whether the cluster of postsynaptic AChRs in the muscle membrane undergoes a similar "plastic" change in its distribution, and how this change occurs. Our results provide information about the mechanisms of postjunctional adaptation occurring during periods of synaptic remodeling in the adult animal in vivo. They suggest that the growing nerve terminal can induce a considerable degree of plasticity in normally stable, postsynaptic AChR clusters.

\section{Materials and Methods}

Female Sprague-Dawley rats weighing 175-200 g were used for these experiments. Injections were performed under direct muscle visualization using a fine 30 gauge needle. Procedures were carried out under chloral hydrate anesthesia $(400 \mathrm{mg} / \mathrm{kg})$.

Botulinum toxin treatment. Crystalline type A botulinum toxin (supplied by Dr. E. Schantz, Food Research Institute, Madison, WI), freshly diluted in mammalian Ringer's solution $\left(1.2 \times 10^{-9} \mathrm{~g}\right.$ in $\left.30 \mu \mathrm{l}\right)$ was injected directly into the surgically exposed soleus muscle.

Surgical denervation. Soleus muscles were denervated by surgically avulsing the sciatic nerve in the thigh.

$\alpha$-Bungarotoxin labeling of AChRs in vivo. $\alpha$-Bungarotoxin ( $\alpha$-BTx), $2 \mu \mathrm{g}$, dissolved in $30 \mu \mathrm{l}$ of Ringer's solution with $1 \% \mathrm{BSA}$, was injected directly into the surgically exposed soleus muscle.

Study of preexisting junctional AChRs during NTO. AChRs at NMJs are thought to be divided into stable and rapidly turning over subpopulations (Stanley and Drachman, 1983). To study the behavior, during NTO, of preexisting "stable" junctional AChRs, i.e., those with a long half-life, we labeled AChRs with $\alpha$-BTx $3 \mathrm{~d}$ before the administration of botulinum toxin. Using this procedure $\alpha$-BTx labeling of AChRs occurs within the 6-12 hr after injection (Stanley and Drachman, 1983). Six days then elapsed between labeling of AChRs and the onset of NTO, which begins about $3 \mathrm{~d}$ after botulinum trcatment. During these $6 \mathrm{~d}$ virtually all ( $>99 \%$ ) of the rapidly turning over AChRs are degraded (half-life, 18-20 hr) or stabilized (Stanley and Drachman, 1983). Thus, at the onset of NTO, virtually all of the $\alpha$-BTx-labeled AChRs at the NMJ are stable. Optimally, in the study of prelabeled AChRs, the initial injection of labeled $\alpha$-BTx (to bind and identify preexisting AChRs) would be followed by perfusion of unlabeled $\alpha$-BTx to prevent rebinding of the original labeled toxin to other AChRs. Unfortunately, this pro- 
tocol was impossible in these experiments because continuous $\alpha$-BTx blockade prevents the NTO phenomenon under study (Pestronk and Drachman 1978b, 1985a). However, previous data from our laboratory and others shows that $\alpha$-BTx does not dissociate from AChRs or rebind to other AChRs to any significant degree (Heinemann et al., 1978; Stanley and Drachman, 1983).

$\alpha-B T x$ labeling of $A C h R s$ in vitro. Air-dried, longitudinal cryostat sections (see below) of frozen muscle were incubated for $1 \mathrm{hr}$ in $0.2 \mu \mathrm{g}$ / $\mathrm{ml} \alpha$-BTx dissolved in Ringer's with 1\% BSA, washed in PBS, and stained as below.

Immunocytochemical staining of AChRs. Soleus muscles were removed, pinned at resting length, and frozen in isopentane cooled with solid $\mathrm{CO}_{2}$. The muscles were sectioned longitudinally (50 $\mu \mathrm{m}$ thickness) in a cryostat. After the sections were air-dried, endogenous peroxidases were removed by incubation with $0.3 \% \mathrm{H}_{2} \mathrm{O}_{2}$ in PBS for $30 \mathrm{~min}$. After this and each subsequent step, sections were washed in PBS. Each antiserum was diluted in PBS with $1 \%$ normal goat serum (NGS). To stain $\alpha$-BTx-labeled AChRs, sections were (1) covered with a $5 \%$ solution of NGS in PBS for 30 min, drained, and covered for $1 \mathrm{hr}$ with rabbit anti$\alpha$-BTx antiserum (1:200); (2) incubated with goat anti-rabbit IgG antiserum $(1: 20) ;(3)$ treated for $1 \mathrm{hr}$ with rabbit peroxidase-antiperoxidase (PAP) (1:40); (4) rinsed in $0.05 \mathrm{~m}$ Tris buffer, $\mathrm{pH} 7.3$, and developed for peroxidase in the Tris buffer using diaminobenzidine (DAB) as the substrate (Sternberger, 1979). Using this technique, the distribution of junctional $\mathrm{AChRs}$ was clearly delineated even in muscles with significant levels of extrajunctional AChRs.

Staining of cholinesterase. In some sections the junctional AChRs and cholinesterase were visualized simultaneously to provide a direct comparison of the distributions of the 2 endplate molecules. In this case, prior to incubation with $\mathrm{H}_{2} \mathrm{O}_{2}$, sections were stained using a bromoindoxylacetate method (Pestronk and Drachman, 1978a), which gives a blue color contrasting with the brown peroxidase-stained AChRs.

Measurement of junctional AChR cluster length. Stained slides were coded and examined "blind," the microscopist having no knowledge of the experiment treatment performed on the muscle. The length of each junction, as outlined by the brown peroxidase stain for AChRs or the blue stain for cholinesterase, was measured parallel to the length of the fiber. This measurement was reliable and reproducible. Similar results were found when repeated counts were made by the same or different examiners.

Quantitation of junctional AChRs. Junctional AChRs were labeled and measured using an ${ }^{125} \mathrm{I}-\alpha$-BTx binding method, as previously described (Berg et al., 1972; Pestronk and Drachman, 1985b). To measure AChRs at elongated junctions, rat soleus muscles were removed and incubated with ${ }^{125} \mathrm{I}-\alpha$-BTx at 3 months after treatment with botulinum toxin. 'T he 3 month period was chosen to allow an accurate quantitative determination of AChRs/NMJ. At this time the number of extrajunctional AChRs has returned to background levels. At earlier times after botulinum treatment, high levels of extrajunctional AChRs make measurement of AChRs/NMJ difficult.

Distribution of newly inserted junctional AChRs. In these experiments, following botulinum treatment, existing AChRs were blocked using unlabeled $\alpha$-BTx (20 $\mu \mathrm{l} \alpha$-BTx in $100 \mu \mathrm{g} / \mathrm{ml}$ in Ringer) injected directly into the soleus muscle. The newly inserted junctional AChRs were studied from 1 to $6 \mathrm{~d}$ later.

In some muscles, an avidin-biotin immunocytochemical technique (Hsu et al., 1981a, b) was used to demonstrate newly inserted AChRs at NMJs. Blockade of existing AChRs was performed $1 \mathrm{~d}$ after botulinum treatment and muscles removed $6 \mathrm{~d}$ later. Longitudinal cryostat sections, $40 \mu \mathrm{m}$ thick, were air-dried, washed in PBS, and lightly fixed with $1 \%$ formaldehyde in PBS for $5 \mathrm{~min}$. After this and each subsequent step, sections were washed in PBS. The sections were sequentially incubated in (1) $0.1 \%$ phenylhydrazine in PBS for $1 \mathrm{hr}$ at $37^{\circ} \mathrm{C}$ to inhibit endogenous peroxidases; (2) custom biotinylated $\alpha$-BTx (Vector Laboratory) at a 1:50 dilution in PBS with $0.1 \%$ BSA for $1 \mathrm{hr}$; (3) cell sortergrade fluorescent-tagged avidin (Vector Laboratory) at a 1:200 dilution in PBS with $0.1 \%$ BSA for $1 \mathrm{hr}$; (4) 5\% NGS in PBS for $15 \mathrm{~min}$ followed by biotinylated goat anti-avidin IgG, 1:200 in PBS with 1\% NGS for 1 hr; and (5) avidin-biotin-peroxidase complex (Vector Laboratory) for $1 \mathrm{hr}$. After washing in PBS and rinsing in $0.05 \mathrm{~m}$ Tris buffer, the sections were developed for peroxidase using $\mathrm{DAB}$ as the substrate. Cobalt chloride, $1 \%$, was added to the DAB solution to help enhance specific staining and reduce background staining (Hsu and Soban, 1982). With immunohistochemistry, the high-density AChR clusters at NMJs were clearly delineated, even in muscles with extrajunctional AChRs.
We used autoradiography to quantitate the distribution of newly inserted junctional AChRs. Blockade of existing junctional AChRs with unlabeled $\alpha$-BTx was performed $6 \mathrm{~d}$ after treatment with botulinum toxin. One day later, $30 \mu \mathrm{l}$ of ${ }^{125} \mathrm{I}-\alpha-\mathrm{BTx}(20 \mu \mathrm{g} / \mathrm{ml})$ was injected into the same soleus muscle. In some cases (when muscles were to be removed $24 \mathrm{hr}$ later), an injection of $20 \mu \mathrm{g}$ of unlabeled $\alpha$-BTx was given $2-3 \mathrm{hr}$ after the ${ }^{125} \mathrm{I}-\alpha$-BTx to prevent further labeling. At $2-24 \mathrm{hr}$ after ${ }^{125} \mathrm{I}-\alpha$-BTx labeling, muscles were removed, frozen, and sectioned longitudinally $(40 \mu \mathrm{M})$ in a cryostat. Sections were washed, stained for cholinesterase using the bromoindoxylacetate method, and processed for autoradiography using Kodak NTB-2 emulsion.

To quantitate the distribution of newly inserted AChRs at elongated NMJs, we compared the density of silver grains at the center and ends of endplates. Endplates were selected for analysis if they were stained en face and were probably elongated ( $62.5 \mu \mathrm{m}$ or longer in length). Counts of grains were made in $12.5 \mu \mathrm{m}$ lengths at the ends and around the center of the cholinesterase-stained endplate. Background grain density, determined on emulsion outside the fibers, was subtracted from all values determined over the fibers.

Statistical methods. Results are expressed as means $\pm \mathrm{SE}$. The significance of the differences between experimental groups was calculated by Student's 2 -tailed $t$-test.

\section{Results}

\section{Changes in the junctional AChR cluster during terminal sprouting}

As in our previous studies, there was a significant amount of nerve terminal outgrowth in rat soleus muscles $7 \mathrm{~d}$ after treatment with botulinum toxin. During this period the nerve terminal arborization became more branched and the patch of junctional cholinesterase elongated. Our results showed that the junctional AChR clusters also became elongated (Fig. 1, $a, b$ ) during the period of NTO. One week after botulinum treatment, junctional AChR clusters in the soleus muscle measured $51 \perp$ $1 \mu \mathrm{m}$ in length, significantly $(p<0.01)$ greater than the $39 \pm$ $0.5 \mu \mathrm{m}$ cluster length in controls (Table 1). In contrast, there was no change in cluster length $(39 \pm 0.8 \mu \mathrm{m})$ a week after surgical denervation of the muscle. The mean lengths of junctional AChR clusters and cholinesterase-stained endplates were similar, both in control muscles and in those treated with botulinum. At individual NMJs the distributions of AChRs and of cholinesterase were virtually identical, with colocalization of the 2 molecules both at control NMJs and at elongated NMJs from muscles treated with botulinum toxin. Elongation of the junctional AChR cluster was accompanied by an increase in the number of AChRs in the cluster. After NTO there were $5.5 \pm$ $0.3 \times 10^{7} \mathrm{AChRs} / \mathrm{NMJ}$ compared with $3.4 \pm 0.4 \times 10^{7} \mathrm{AChRs} /$ NMJ $(p<0.001)$ in control soleus muscles, a $62 \%$ increase.

\section{Mechanisms underlying the growth of the junctional AChR cluster}

New junctional AChRs. We first examined whether AChRs that were newly synthesized and inserted in the muscle membrane play a role in the elongation of the junctional AChR cluster. New AChRs were studied by blocking preexisting AChRs with $\alpha$-BTx, waiting $24 \mathrm{hr}$ to $6 \mathrm{~d}$ for new AChRs to be inserted into the membrane, and then selectively labeling the new AChRs with ${ }^{125} \mathbf{I}-\alpha$-BTx or biotin- $\alpha$-BTx. Our results showed that in botulinum-treated muscles new AChRs were present over the entire length of elongated NMJs. The distribution of new AChRs at NMJs was similar to that of the total population of junctional AChRs and to the pattern of cholinesterase at both normal and clongatcd NMJs (Fig. 2, $a, b$ ). The intensity of staining of new junctional AChRs was relatively uniform over the NMJ; there 
Table 1. Effects of botulinum treatment and denervation on the length of the junctional $\mathrm{AChR}$ cluster and the cholinesterase patch

\begin{tabular}{lll} 
Treatment & $\begin{array}{l}\text { Junctional } \\
\text { AChR cluster } \\
(\mu \mathrm{m})\end{array}$ & $\begin{array}{l}\text { Junctional } \\
\text { cholinesterase } \\
\text { patch } \\
(\mu \mathrm{m})\end{array}$ \\
\hline $\begin{array}{l}\text { Botulinum } \\
\text { Control }\end{array}$ & $51 \pm 1^{a}(8)$ & $52 \pm 3^{a}(6)$ \\
$\begin{array}{l}\text { Denervation } \\
\text { Control }\end{array}$ & $39 \pm 0.5(8)$ & $40 \pm 2(7)$ \\
\hline
\end{tabular}

Botulinum-treated muscles were examined after $7 \mathrm{~d}$ when NTO was abundant. Denervated muscles were examined $7 \mathrm{~d}$ after sciatic nerve avulsion. Results are expressed as means \pm SE. Numbers in parentheses indicate number of muscles sampled. Note that botulinum treatment caused similar elongations of AChR cluster and cholinesterase patches. In contrast, denervation caused no change in the length of $\mathrm{AChR}$ clusters.

${ }^{a}$ Greater than control, $p<0.001$.

were no consistent differences between the center and edges of NMJs. Autoradiography confirmed this impression. AChRs newly inserted during the $1 \mathrm{~d}$ period from 6 to $7 \mathrm{~d}$ after botulinum treatment were distributed evenly over the length of the NMJ, as demarcated by the cholinesterase stain. Counts of silver grains were similar over central and end segments of elongated NMJs (Table 2).

Redistribution of junctional AChRs. We next studied the behavior of preexisting junctional AChRs during growth of the NMJ (Fig. 3, $a, b$ ). AChRs were labeled in vivo by injecting $\alpha$-BTx into the soleus muscle $3 \mathrm{~d}$ before botulinum treatment. Seven days after botulinum treatment the distribution of the remaining $\alpha$-BTx-labeled AChRs was compared to the total population of AChRs at NMJs in botulinum-treated and control muscles. Our results showed that the distribution of the preexisting stable junctional AChRs changed during growth of the NMJ. The average length of clusters of preexisting junctional AChRs was $53 \pm 2 \mu \mathrm{m}$ at $7 \mathrm{~d}$ after botulinum treatment, significantly $(p<0.01)$ greater than the $44 \pm 1 \mu \mathrm{m}$ for controls. Histograms also demonstrated the increase in the length of preexisting junctional AChR clusters after NTO (Fig. 4). At individual endplates the distribution of preexisting AChRs mirrored that of cholinesterase even at the most elongated NMJs.

Migration of extrajunctional AChRs. In a final set of experiments we examined whether migrating extrajunctional AChRs contribute to the elongated junctional AChR cluster. In developing muscle fibers this phenomenon has been documented by labeling extrajunctional $\mathrm{AChRs}$ and finding them to be concentrated at NMJs, especially around their periphery, 1-3 d later (Anderson and Cohen, 1977; Weinberg et al., 1981; Stya and Axelrod, 1983; Ziskind-Conhaim et al., 1984; Role et al., 1985). In the present studies we blocked preexisting AChRs in the soleus muscle $6 \mathrm{~d}$ after treatment with botulinum toxin. The AChRs that accumulated in the membrane during the next 24 $\mathrm{hr}$ were then labeled with ${ }^{125} \mathrm{I}-\alpha$-BTx. Two hours afterward an injection of 40 -fold more $\alpha$-BTx was injected to inhibit further ${ }^{125} \mathrm{I}-\alpha$-BTx labeling of AChRs. Two and 24 hours after labeling with ${ }^{125} \mathrm{I}-\alpha-\mathrm{BT}$, we measured the pattern of labeled AChRs at the NMJ. Our data showed that at both time points labeled AChRs were distributed evenly over the length of the NMJ (Table 2). There was no evidence of accumulation of labeled AChRs at the periphery of the NMJ. Grain density at NMJs decreased by $13 \%$ over the $22 \mathrm{hr}$ period.
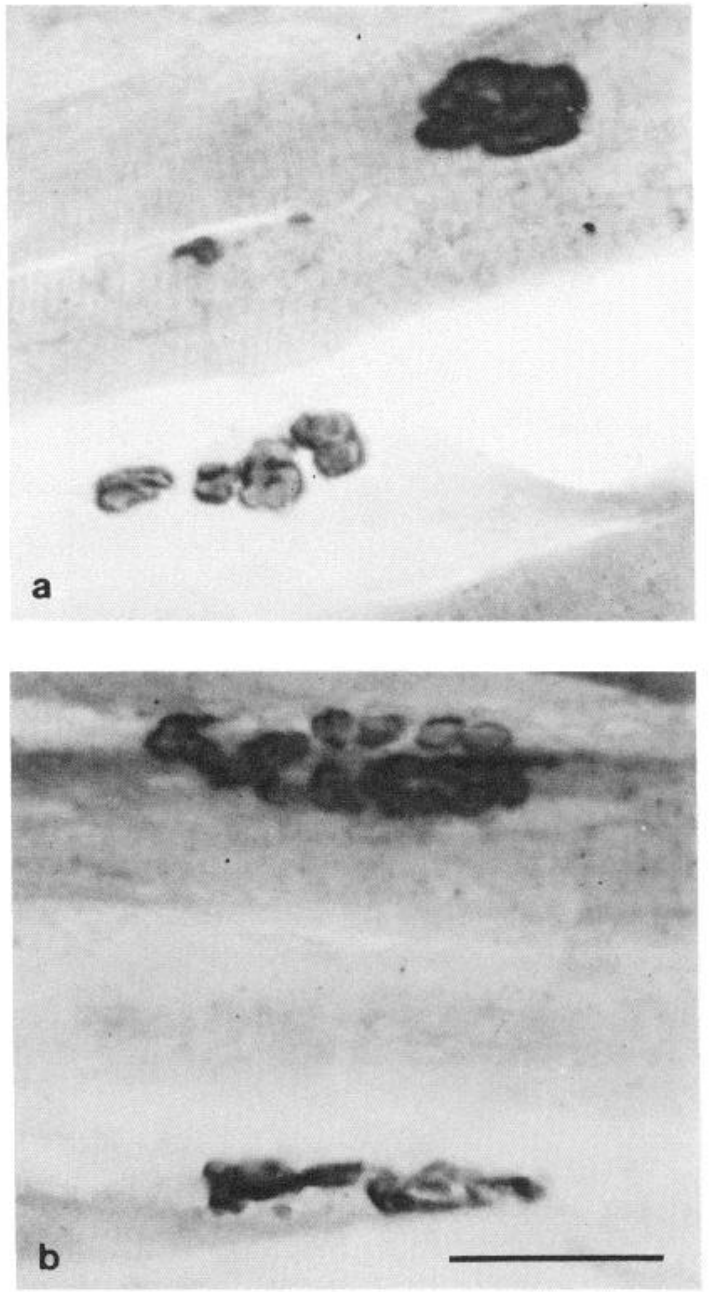

Figure 1. Junctional AChR clusters in rat soleus muscles stained immunocytochemically. $a$, Junctional AChR clusters from normal muscle. $b$, Junctional AChR clusters from muscle $7 \mathrm{~d}$ after treatment with botulinum toxin. There is significant elongation of cluster lengths. Bar, 50 $\mu \mathrm{m}$.

\section{Discussion}

\section{Association of NTO with AChR cluster elongation}

In the normal adult animal the cluster of postsynaptic junctional AChRs is remarkably stable. The turnover of most of these AChRs is slow (Berg and Hall, 1975; Chang and Huang, 1975; Fambrough, 1979; Levitt and Salpeter, 1981), they have limited mobility within the muscle membrane (Fambrough and Pagano, 1977; Axelrod et al., 1979; Salpeter and Harris, 1983), and their total number persists relatively unchanged throughout the life of the animal (Pestronk et al., 1980). In the present study we have found that NTO can induce rapid modifications in this normally stable junctional AChR cluster. During such periods of synaptic plasticity (i.e., NTO), the junctional AChR cluster undergoes distinct changes, becoming elongated and accumulating increased numbers of AChRs.

The change in the distribution of junctional AChRs during NTO parallels the remodeling of other synaptic elements. Quantitatively, the increase in the mean length of the junctional $\mathrm{AChR}$ cluster is similar to that of the cholinesterase stained at the NMJ. At individual NMJs, both in normals and after NTO, the pattern of the junctional AChR cluster in muscle membrane is virtually 

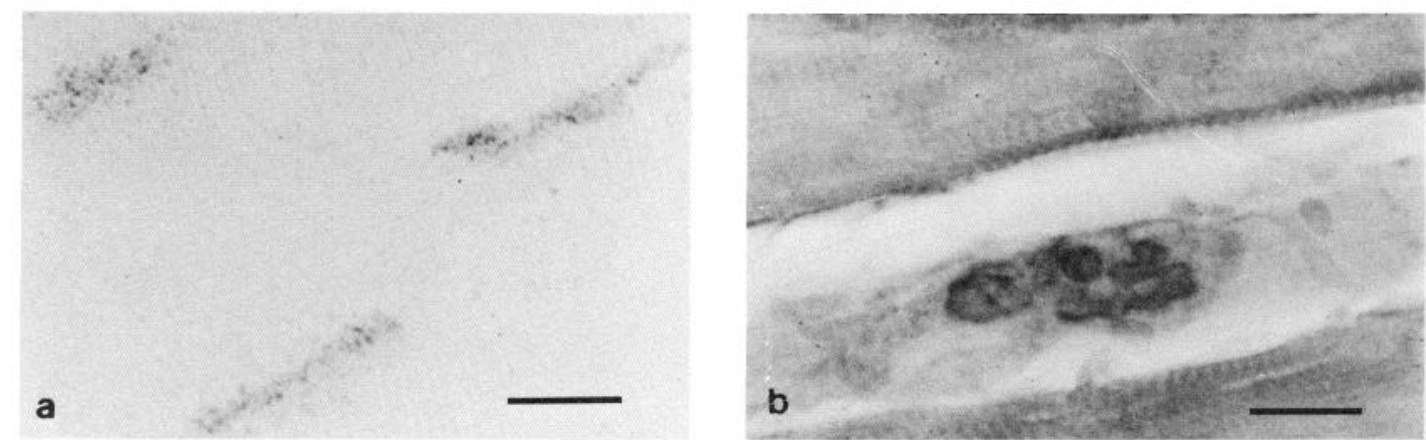

Figure 2. New junctional AChRs appearing during a period of NTO. $a$, Autoradiogram of new AChRs appearing after botulinum treatment. New $\mathrm{AChRs}$ inserted between days 6 and 7 after botulinum treatment were labeled in vivo with ${ }^{125} \mathrm{I}-\alpha-\mathrm{BTx}$ and muscles removed $2 \mathrm{hr}$ later. Endplates are also lightly stained for cholinesterase. Note the even distribution of silver grains at NMJs. Bar, $40 \mu \mathrm{m} . b$, NMJ stained for new AChRs appearing after botulinum treatment, using immunocytochemistry. The staining is uniformly distributed over the length of the NMJ. Bar, $25 \mu \mathrm{m}$.

identical to that of cholinesterase in the basal lamina, which, in turn, mirrors the distribution of the presynaptic nerve terminal arborization. The close anatomic correspondence between junctional AChRs, cholinesterase, and nerve terminals, even during periods of the modification of the NMJ, suggests the existence of precise interactions among these synaptic elements.

\section{Mechanisms of AChR cluster growth}

In the present experiments we have studied synaptic plasticity at NMJs treated with botulinum toxin. Botulinum treatment blocks neuromuscular transmission (Brooks, 1954; Kao et al., 1976; Simpson, 1981) resulting in denervation-like changes in muscle which, in turn, stimulate NTO (Duchen and Stritch, 1968; Brown et al., 1981; Pestronk and Drachman, 1985a). One could postulate that the change in the junctional AChR cluster occurring after botulinum treatment is either (1) a direct byproduct of denervation changes in muscle such as fiber atrophy or an increase in extrajunctional AChRs or (2) induced by NTO. However, the former possibility seems unlikely. After nerve section, a procedure that produces more denervation changes in muscle than botulinum treatment (Mathers and Thesleff, 1978; Drachman et al., 1982), there is no growth of the junctional $\mathrm{AChR}$ cluster. It thus seems probable that the growth in the junctional AChR cluster seen in the present experiments requires the anatomical integrity of the nerve and is secondary to the process of NTO.

The NTO-induced growth of junctional AChR clusters could

\section{Table 2. Distribution of new AChRs at elongated NMJS}

\begin{tabular}{lll} 
& \multicolumn{2}{l}{ Silver grains $/ \mu \mathrm{m}$} \\
\cline { 2 - 3 } NMJ segment & $2 \mathrm{hr}$ after label & $24 \mathrm{hr}$ after label \\
\hline End & $7.7 \pm 0.2$ & $6.7 \pm 0.3$ \\
Center & $7.9 \pm 0.3$ & $6.9 \pm 0.4$
\end{tabular}

The uniformity of distribution of newly inserted AChRs at elongated NMJs was examined by comparing the density of AChRs in central and peripheral endplate segments. Existing AChRs in soleus muscles were blocked with unlabeled $\alpha$-BTx at $6 \mathrm{~d}$ after botulinum treatment. One day later, new AChRs were labeled in vivo with ${ }^{125} \mathrm{I}-\alpha$-BTx. Muscles were removed 2 and $24 \mathrm{hr}$ after labeling and autoradiograms obtained of longitudinal frozen sections. At each NMJ, silver grains were counted over $12.5 \mu \mathrm{m}$ lengths at the ends and $25 \mu \mathrm{m}$ lengths at the centers of the NMJ. Counts are expressed as number of silver grains per micron of NMJ length. Note that the distribution of grains is even. There is no significant difference at either time point between the density of labeled AChRs at the ends and centers of the NMJ. occur by several mechanisms, including (1) insertion of newly synthesized AChRs into the membrane at the NMJ, either focally to form new areas of AChR clustering or generally over the whole length of the longer postsynaptic membrane; (2) mi-
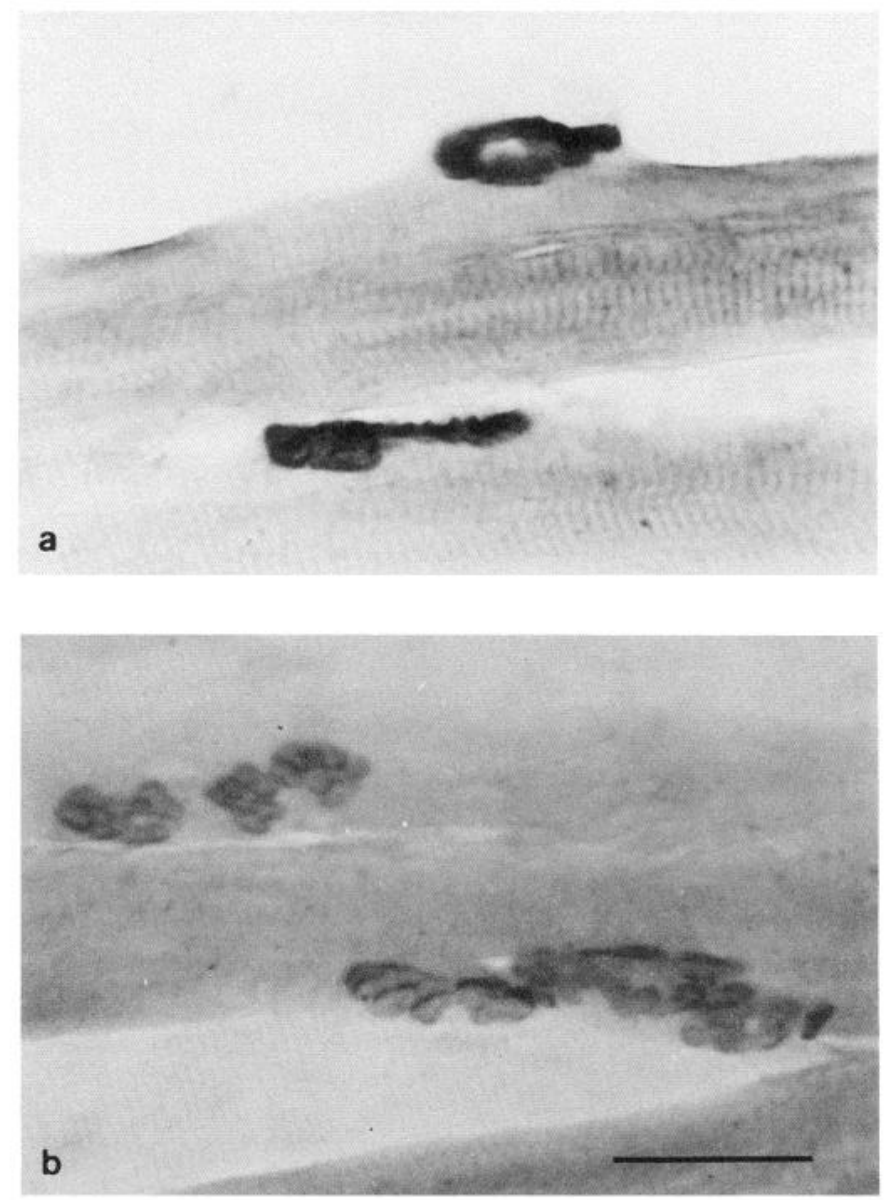

Figure 3. Preexisting junctional AChRs in rat soleus muscles stained immunocytochemically. $a$, Preexisting junctional AChRs from normal muscle. Muscles were labeled in vivo $3 \mathrm{~d}$ earlier with $\alpha$-BTx. $b$, Preexisting junctional $\mathrm{AChRs}$ after botulinum treatment. Muscles were labeled with $\alpha$-BTx in vivo $3 \mathrm{~d}$ prior to treatment with botulinum toxin and stained another $7 \mathrm{~d}$ later. Note significant elongation of cluster lengths compared with controls. Bar, $50 \mu \mathrm{m}$. 
DISTRIBUTION OF 'PRE-EXISTING' AChR CLUSTER LENGTHS BEFORE AND AF TER BOTULINUM TREATMENT

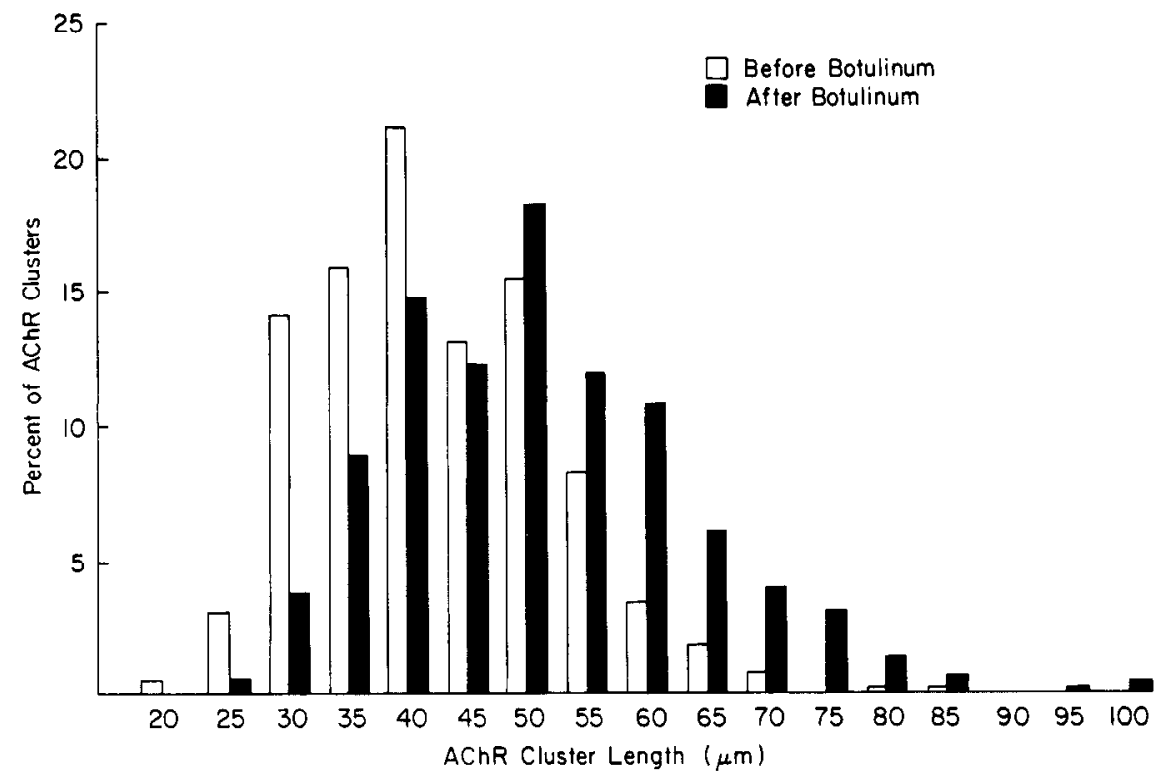

Figure 4. Lengths of clusters of preexisting AChRs in botulinum-treated and control muscles. "Preexisting" junctional AChRs were labeled with $\alpha$-BTx in vivo $3 \mathrm{~d}$ before treatment of soleus muscle with botulinum toxin. The muscles were removed and studied another $7 \mathrm{~d}$ later. Control muscles were labeled with $\alpha$-BTx but not treated with botulinum. At least $40 \mathrm{AChR}$ clusters per muscle were measured. The histograms were drawn using $436 \mathrm{AChR}$ clusters from 8 botulinum-treated muscles and 412 AChR clusters from 8 control muscles. Note the increased population of longer junctional AChR clusters in botulinum-treated muscles. The mean length of clusters of "preexisting" junctional AChRs was $53 \pm 2 \mu \mathrm{m}$ for botulinum-treated muscles compared with $44 \pm 1 \mu \mathrm{m}$ for control muscles. Thus, preexisting $A C h R s$ are redistributed during NMJ elongation. gration of extrajunctional AChRs to form new areas of $\mathrm{AChR}$ clustering or to add AChRs to the preexisting cluster; and (3) redistribution of old or preexisting junctional $\mathrm{AChRs}$ over a longer length of muscle membrane.

Our results show that new AChRs are added to the entire length of the junctional AChR cluster. Autoradiograms of muscle fibers $24 \mathrm{hr}$ after blockade of preexisting AChRs showed the selective accumulation of new AChRs at normal and elongated NMJs. Eventually this process leads to a $62 \%$ increase in junctional AChRs in muscles with elongated NMJs compared to normal controls.

AChRs, newly inserted at enlarging NMJs in botulinum-treated muscles, could derive either from the population of newly synthesized AChRs or from extrajunctional AChRs migrating to the NMJ. In developing muscle fibers, preexisting extrajunctional AChRs can migrate in the membrane to form a cluster at the site of a new nerve-muscle contact (Anderson and Cohen, 1977; Stya and Axelrod, 1983; Ziskind-Conhaim et al., 1984; Role et al., 1985). Migration of extrajunctional AChRs may also contribute to endplates forming in ectopically innervated muscle (Weinberg et al., 1981). At preformed NMJs in adult muscle one would expect such migrating AChRs to accumulate preferentially around the periphery of the NMJ. However, in the present experiments new AChRs accumulated uniformly over the length of elongated NMJs. There was no evidence of selective localization of new junctional AChRs at the periphery of NMJs. This suggests that, if migration of extrajunctional AChRs to the NMJs occurs, it provides only a minority of the new junctional AChRs. In any case, at later times (e.g., 3 months after botulinum treatment), when extrajunctional $A C h R s$ have virtually disappeared, redistributed receptors cannot provide the increased supply of AChRs at elongated NMJs.

A final source of AChRs at elongated NMJs could be redistribution of preexisting NMJ AChRs. At first consideration this possibility seems unlikely because, as noted above, junctional AChRs have been thought to be very stable. However, our data show that NTO can induce significant movement in the previously stable AChRs. After NTO-induced elongation of the
$\mathrm{NMJ}$, the preexisting stable junctional $\mathrm{AChRs}$ are redistributed over the entire new length of the NMJ. This suggests that NTO can precisely direct the movement of AChRs over distances of up to $15-30 \mu \mathrm{m}$ in the skeletal muscle membrane. The exact pattern of receptor movement is unclear. Possibilities include migration of individual AChR molecules, movement or elongation of some of the subdivisions of AChRs that are often found at mammalian NMJs (Figs. 1, 3) (Salpeter and Harris, 1983), or reorganization of the entire NMJ.

In conclusion, the present study provides further documentation of the remarkable and complex bidirectional trophic interactions between motor nerves and muscle AChRs. In adult muscles, with normal synaptic transmission, $\mathrm{AChRs}$ in muscle are localized at its site of contact with the motor nerve. When synaptic transmission from nerve to muscle is interrupted, new AChRs appear all along the muscle membrane. These muscle AChRs have a reverse trophic influence, inducing outgrowth from the presynaptic nerve terminals (Pestronk and Drachman, 1978b, 1985a). We have now found that the muscle-induced outgrowth of presynaptic nerve terminals, in turn, causes elongation of the AChR cluster at the NMJ. Our data suggest that mechanisms in skeletal muscle underlying this plasticity of the postsynaptic membrane include redistribution of preexisting AChRs and synthesis of new AChRs. Further studies are required to understand the trophic influence from nerve that can induce such anatomically precise changes in a previously stable postsynaptic membrane.

\section{References}

Anderson, M. J., and M. W. Cohen (1977) Nerve-induced and spontaneous redistribution of acetylcholine receptors on cultured muscle cells. J. Physiol. (Lond.) 268: 757-773.

Anderson, M. J., M. W. Cohen, and E. Zorychta (1977) Effects of innervation on the distribution of acetylcholine receptors on cultured muscle cells. J. Physiol. (Lond.) 268: 731-756.

Axelrod, D., P. Ravdin, D. E. Koppel, J. Schlessinger, M. W. Webb, E. L. Elson, and T. R. Podleski (1979) Lateral motion of fluorescently labelled acetylcholine receptors in membranes of developing muscle fibers. Proc. Natl. Acad. Sci. USA 73: 4594-4598. 
Berg, D. K., and Z. W. Hall (1975) Loss of $\alpha$-bungarotoxin from junctional and extrajunctional acetylcholine receptors in rat diaphragm muscle in vivo and in organ culture. J. Physiol. (Lond.) 252: $771-789$.

Berg, D. K., R. B. Kelly, P. B. Sargent, P. Williamson, and Z. W. Hall (1972) Binding of $\alpha$-bungarotoxin to acetylcholine receptors in mammalian muscle. Proc. Natl. Acad. Sci. USA 69: 147-151.

Bevan, S., and J. H. Steinbach (1977) The distribution of $\alpha$-bungarotoxin sites of mammalian skeletal muscle developing in vivo. J. Physiol. (Lond.) 267: 195-213.

Braithwaite, A. W., and A. J. Harris (1979) Neural influence on acetylcholine receptors in embryonic development of skeletal muscle. Nature 276: 549-551.

Brooks, V. B. (1954) The action of botulinum toxin on motor nerve filaments. J. Physiol. (Lond.). 123: 501-515.

Brown, M. C., R. L. Holland, and W. B. Hopkins (1981) Motor nerve sprouting. Annu. Rev. Neurosci. 4: 17-42.

Burden, S. (1977) Development of the neuromuscular junction in the chick embryo: The number, distribution and stability of acetylcholine receptors. Dev. Biol. 57: 317-329.

Chang, C. C., and M. C. Huang (1975) Turnover of junctional and extrajunctional receptors of the rat diaphragm. Nature 253: 643-644.

Drachman, D. B., E. F. Stanley, A. Pestronk, J. W. Griffin, and D. L. Price (1982) Neurotrophic regulation of two properties of skeletal muscle by impulse-dependent and spontaneous acetylcholine transmission. J. Neurosci. 2: 232-243.

Duchen, I. W., and S. J. Stritch (1968) The effects of botulinum toxin on the pattern of innervation of skeletal muscle in the mouse. Q. J. Exp. Physiol. 53: 84-89.

Fambrough, D. M. (1974) Acetylcholine receptors: Revised estimates of extrajunctional receptor density in denervated rat diaphragm. $\mathbf{J}$. Gen. Physiol. 64: 468-472.

Fambrough, D. M. (1979) Control of acetylcholine receptors in skeletal muscle. Physiol. Rev. 59: 165-227.

Fambrough, D. M., and H. C. Hartzell (1972) Acetylcholine receptors: Number and distribution at neuromuscular junctions in rat diaphragm. Science 176: 189-191.

Fambrough, D. M., and R. E. Pagano (1977) Positional stability of acetylcholine receptors at the neuromuscular junction. Carnegie Inst. Washington Yearb. 76: 28-29.

Fertuck, H. C., and M. M. Salpeter (1976) Quantitation of junctional and extrajunctional acetylcholine receptors by E.M. autoradiography after ${ }^{125} \mathrm{I}-\alpha$-bungarotoxin binding at mouse neuromuscular junctions. J. Cell Biol. 69: 144-158.

Frank, E., and G. D. Fischbach (1979) Early events in neuromuscular junction formation in vitro. J. Cell Biol. 83: 143-158.

Hartzell, H. C., and D. M. Fambrough (1972) Acetylcholine receptors: Distribution and extrajunctional density in rat diaphragm after denervation correlated with acetylcholine sensitivity. J. Gen. Physiol. 60: $248-262$

Heinemann, S., J. Merlie, and J. Lindstrom (1978) Modulation of acetylcholine receptor in rat diaphragm by anti-receptor sera. Nature 274: 65-68.

Hsu, S.-M., and E. Soban (1982) Color modification and diaminobenzidine (DAB) precipitation by metallic ions and its application for double immunohistochemistry. J. Histochem. Cytochem. 30: 10791082.

Hsu, S.-M., L. Raine, and H. Fanger (1981a) Use of avidin-biotinperoxidase complex $(\mathrm{ABC})$ in immunoperoxidase techniques. J. Histochem. Cytochem. 29: 577-580.
Hsu, S.-M., L. Raine, and H. Fanger (1981b) The use of antiavidin antibody and avidin-biotin-peroxidase complex in immunoperoxidase techniques. A. J. Clin. Pathol. 75: 816-821.

Kao, I., D. B. Drachman, and D. L. Price (1976) Botulinum toxin: Mechanism of presynaptic blockadc. Scicncc 193: 1256-1258.

Levitt, T. A., and M. M. Salpeter (1981) Denervated endplates have a dual population of junctional acetylcholine receptors. Nature 291 : 239-241.

Mathers, D. A., and S. Thesleff (1978) Studies on neurotrophic regulation of murine skeletal muscle. J. Physiol. (Lond.) 282: 105-114.

Miledi, R. (1960) The acetylcholine sensitivity of frog muscle fibers after complete or partial denervation. J. Physiol. (Lond.) 151: 1-23.

Pestronk, A., and D. B. Drachman (1978a) A new stain for quantitative measurement of sprouting at neuromuscular junctions. Muscle Nerve 1: 70-74.

Pestronk, A., and D. B. Drachman (1978b) Motor nerve sprouting and acetylcholine receptors. Science 199: 1223-1225.

Pestronk A., and D. B. Drachman (1985a) Motor nerve terminal outgrowth and acetylcholinc rcceptors: Inhibition of terminal outgrowth by $\alpha$-bungarotoxin and anti-acetylcholine receptor antibody. J. Neurosci. 5: 751-758.

Pestronk, A., and D. B. Drachman (1985b) Measurement of junctional acetylcholine receptors in myasthenia gravis: Clinical correlates. Muscle Nerve 8: 245-251.

Pestronk, A., D. B. Drachman, and J. W. Griffin (1976) Effect of botulinum toxin on trophic regulation of acetylcholine receptors. $\mathrm{Na}$ ture 264: 787-789.

Pestronk, A., D. B. Drachman, and J. W. Griffin (1980) Effects of aging on sprouting and regeneration. Exp. Neurol. 70: 62-85.

Role, L. W., V. R. Matossian, R. J. O'Brien, and G. D. Fischbach (1985) On the mechanism of acetylcholine receptor accumulation at newly formed synapses on chick myotubes. J. Neurosci. 5: 2197-2204.

Salpeter, M. M., and R. Harris (1983) Distribution and turnover rate of acetylcholine receptors throughout the junction folds at a vertebrate neuromuscular junction. J. Cell. Biol. 96: 1781-1785.

Simpson, L. L. (1981) The origin, structure and pharmacological activity of botulinum toxin. Pharmacol. Rev. 33: 155-188.

Slater, C. R. (1982) Neural influence on postnatal changes in acetylcholine receptor distribution in nerve-muscle junctions in the mouse. Dev. Biol. 94: 23-30.

Stanley, E. F., and D. B. Drachman (1983) Rapid degradation of "new" acetylcholine receptors at neuromuscular junctions. Science 222: 6769.

Sternberger, L. (1979) Immunocytochemistry, 2nd ed., Chap. 5, Wiley, New York.

Stya, M., and D. Axelrod (1983) Diffusely distributed acetylcholine receptors can participate in cluster formation on cultured rat myotubes. Proc. Natl. Acad. Sci. USA 80: 449-453.

Stya, M., and D. Axelrod (1984) Mobility of extrajunctional acetylcholine receptors on denervated adult muscle fibers. J. Neurosci. 4: 70-74.

Weinberg, C. B., C. G. Reiness, and Z. W. Hall (1981) Topographical segregation of old and new acetylcholine receptors at developing ectopic endplates in adult rat muscle. J. Cell Biol. 88: 215-218.

Young, S. H., and M-M. Poo (1983) Rapid lateral diffusion of extrajunctional acetylcholine receptors in developing muscle membrane of Xenopus tadpole. J. Neurosci. 3: 225-231.

Ziskind-Conhaim I... I. Geffen, and Z. W. Hall (1984) Redistribution of acetylcholine receptors on developing rat myotubes. J. Neurosci. 4: $2346-2349$. 